\title{
Profile of nimotuzumab in the treatment of high-grade glioma
}

\author{
This article was published in the following Dove Press journal: \\ OncoTargets and Therapy \\ 13 April 2015 \\ Number of times this article has been viewed
}

\section{Qun-ying Yang \\ Cheng-cheng Guo \\ Zhong-ping Chen}

Department of Neurosurgery/ Neuro-oncology, Sun Yat-sen

University Cancer Center, Guangzhou,

People's Republic of China
Correspondence: Zhong-ping Chen Department of Neurosurgery/Neurooncology, Sun Yat-sen University Cancer Center, Guangzhou 5I0060, People's Republic of China

Email chenzhp@sysucc.org.cn

\begin{abstract}
High-grade gliomas (HGG) are extremely aggressive lesions and represent the most common primary malignant brain tumors without an effective therapy. Standard treatment for HGG usually includes surgery followed by radiotherapy and chemotherapy. However, the prognosis of patients with HGG remains dismal. We review the humanized epidermal growth factor receptor (EGFR) and the major EGFR target drugs in HGG treatments, focusing on the EGFR antibody nimotuzumab as a new therapeutic strategy in HGG. We found that nimotuzumab with or without radiotherapy, chemotherapy in newly diagnosed or recurrent HGG, such as glioblastoma multiforme (GBM), anaplastic astrocytomas (AA), and diffuse intrinsic pontine glioma (DIPG), might improve the response rate or the survival time. In conclusion, nimotuzumab is a very well-tolerated drug with acceptable toxicity, and it may have promising value in the combination treatment. As a result, multiple center randomized controlled Phase III clinical trials need to be conducted to confirm the efficacy and toxicity for nimotuzumab in HGG.
\end{abstract}

Keywords: nimotuzumab, high-grade gliomas, chemotherapy, radiotherapy

\section{Treatment status and prognosis of HGG}

High-grade gliomas (HGG) are those gliomas of grades III-IV, also known as malignant gliomas, that have extremely aggressive lesions and represent the most common primary malignant brain tumors with an annual incidence of 5.26/100,000/year. Despite remarkable advances in cancer research, the prognosis of HGG is uniformly poor despite advances in treatment. Standard treatment for HGG is surgery followed by radiation and chemotherapy. Temozolomide (TMZ) is indicated for anaplastic astrocytomas (AA) and glioblastoma multiforme (GBM) as a standard chemotherapy drug. However, the prognosis of patients with HGG remains dismal. The 5-year survival rate of newly diagnosed patients with GBM, who received standard concurrent and adjuvant temozolomide chemotherapy is still less than $10 \%{ }^{1,2}$ The poor prognosis of patients with HGG has led to the search for new therapeutic strategies.

\section{EGFR expression in glioma EGFR status in glioma}

Epidermal growth factor receptor (EGFR) is one of the major genetic factors affecting the pathogenesis and prognosis of HGG. EGFR is a membrane-bound receptor that has been shown to have a major role in the pathogenesis and progression of different cancers. ${ }^{3}$ The frequent amplification of the EGFR gene in glioma was initially reported in 1985. Totally, more than half of HGG overexpress EGFR. ${ }^{4}$ It has been confirmed in the aspects of genetic amplification, elevated expression, and mutation. Moreover, EGFR plays an essential role in the pathogenesis of glioma. Mazzoleni presented evidence to show that ${ }^{5}$ cancer stem cells (CSC), which were present in the resistant glioma cells, express EGFR to promote tumorigenesis. EGFR-expressing initiating cells display the most malignant subtype. High 
expression of EGFR protein in glioma has been associated with tumor progression and enhanced tumorigenicity. EGFR is well validated as a primary contributor of HGG initiation and progression. What is more, EGFR overexpression is related to more aggressive disease, resistant to both radiation and chemotherapy, and a poorer prognosis. ${ }^{6}$

With regard to the subtype analysis, EGFR amplification has been reported only in 3\% of anaplastic (grade III) astrocytomas, and it is infrequent in secondary GBMs (only 8\%), whereas $60 \%$ of primary GBMs show EGFR overexpression, and $40 \%$ of them contain EGFR amplifications. Furthermore, EGFR amplification is rare in GBM patients younger than 35 years. EGFRvIII is the most common mutation site (inframe deletion spanning exons 2-7, imparting ligand-independent activation and high oncogenicity), and the EGFRvIII deletion represents $60 \%-70 \%$ of EGFR mutations in GBM, which encodes for a receptor that lacks amino acids 6-273 of the extracellular domain. Studies of the mouse glioma models have indicated that the EGFRvIII variant is more tumorigenic than the wild-type receptor in primary GBMs, EGFR aberrations are accompanied by mutations in tumor suppressors p16Ink4a, p19Arf, and PTEN, constitutively activating several oncogenes like STATs, Akt, Erk1/2, and so on, while secondary GBMs are mostly associated with mutation/deletions in p53, deregulating the cell cycle and also aberrant activation of isocitrate dehydrogenase (IDH) and PDGFR (platelet-derived growth factor receptor) pathway.

\section{EGFR-targeted drugs in glioma}

Gefitinib (ZD1839; Iressa) is a small molecular EGFR kinase inhibitor. It has been approved for locally advanced and metastatic non-small-cell lung cancer (NSCLC). A Phase II clinical trial confirmed the efficacy of gefitinib in 57 recurrent GBM patients. The median EFS (event-free survival) time was 8.1 weeks and the median OS (overall survival) was 39.4 weeks, with $13 \%$ of 6 -month EFS. In another Phase I/II trial, the combination of gefitinib and another mTOR inhibitor, everolimus, did not show promising efficacy. There was only one in 22 patients with PFS beyond 6 months.

1) Erlotinib (OSI-774; Tarceva) is also a small molecule EGFR kinase inhibitor that has been approved to treat metastatic NSCLC. The combination of erlotinib and gemcitabine is effective for local advance or metastatic pancreatic cancer. Several Phase I/II clinical trials have examined the effects of a combination of erlotinib with $\mathrm{TMZ}$ and radiation, but it seemed to have no clear effect in GBM patients.

2) Lapatinib (GW572016; Tykerb/Tyverb) is a small molecule inhibitor targeting both EGFR and Her-2. It has been approved for the treatment of local advanced or metastatic breast cancer, especially for patients with Her-2 positive who have failed to respond to other drugs. The Phase I/II trial did not show efficacy in 24 patients with recurrent GBM. Another Phase II trial (NCT00103129) using lapatinib was recently completed in recurrent GBMs and gliosarcomas, and the final result is awaited.

3) Cetuximab (C225; Erbitux) is a humanized monoclonal EGFR antibody used for metastasized or recurrent colorectal cancer and squamous cell carcinoma of the head and neck. It is also used with radiation therapy, as a firsttime treatment for advanced squamous cell carcinoma of the head and neck. Cetuximab was designed in combination with bevacizumab and irinotecan, but yielded similar benefits to those from the combination of bevacizumab and irinotecan. Further evaluation of this regimen is not planned by the investigators.

4) Panitumumab (ABX-EGF; Vectibix) is a human monoclonal antibody that recognizes the extracellular domain of EGFR. It is used for metastatic colorectal cancer that has failed other therapies. All these agents except panitumumab have been examined in Phases I and II clinical trials in malignant glioma patients. Several anti-EGFR-based therapeutic strategies have been assessed in preclinical and clinical trials as a single drug or in combination with radiotherapy and conventional chemotherapy. However, these EGFR-targeted drugs suggested relatively insufficient in HGG.

\section{Resistance of the EGFR-targeted therapy}

It was difficult to reconcile the efficacy of the EGFR-targeted drugs with the perceived importance of the EGFR target in HGG. There was crosstalk pathway for inhibition of EGFR. The expression of the constitutively active mutant EGFRvIII could sensitize tumors to EGFR inhibitors, whereas PTEN loss would impair the response to such inhibitors. It has also been proved that high levels of EGFR along with low levels of activated Akt, which is an essential effector of PI3K signaling, would predict a favorable response in preclinical studies. Another study showed that EGFR signaling through regulating $\mathrm{PI} 3 \mathrm{~K} / \mathrm{mTOR}$ is critical for mediating the response to EGFR-targeted drugs. As a result, the feedback and cross-over signaling between different targets need to be confirmed to promote responsiveness.

\section{Clinical studies with nimotuzumab}

Nimotuzumab (h-R3) is a humanized IgG1 isotype monoclonal antibody that binds to the extracellular domain of EGFR, inhibiting tyrosine kinase activation. ${ }^{7}$ It has been shown that nimotuzumab could inhibit proliferation, survival, and angiogenesis of gliomas. Nimotuzumab has been approved for the squamous cell carcinoma of head and neck, glioma, and 
nasopharyngeal cancer in different countries. It has also been granted approval for use as an orphan drug status for glioma in the USA and for glioma and pancreatic cancer in Europe. ${ }^{7}$ It can reach CNS tumors in nonclinical and clinical settings. It was determined that the combination of nimotuzumab and radiotherapy significantly increased the inhibition of EGFR-related signaling pathway, increasing the antiproliferative activity of both therapies (Figure 1), ${ }^{8}$ and seemed to be effective in adult and children glioma. ${ }^{9}$ In this article, the clinical trials using nimotuzumab for malignant glioma are reviewed.

\section{Phase I/II trials}

\section{A Phase I/II trial of nimotuzumab in newly diagnosed HGG patients}

In order to determine safety, immunogenicity, and response of nimotuzumab in newly diagnosed HGG patients, Ramos et $\mathrm{al}^{10}$ conducted a multicenter, open-labeled, Phase I/II trial. Patients received 6 weekly infusions of nimotuzumab in
$200 \mathrm{mg}$ doses in combination with external beam radiotherapy with a standard dose of 50-60 Gy. Between February 2002 and February 2004, 29 patients were entered into the study, newly diagnosed with GBM (16 patients), AA (12 patients), and anaplastic oligodendroglioma (AO) (one patient). The antibody was very well tolerated. There were no grade 3/4 adverse toxicities detected. No acneiform rash or allergic reactions were discovered in these patients. The objective response rate was $37.9 \%$, with $17.2 \%$ complete response (CR), 20.7\% partial response (PR), and 41.4\% stable disease (SD). With a median time of 29 months, the median survival time (MST) is 22.17 months for all subjects (17.47 months for GBM, and the MST is not reached for AA patients).

In the trial, the capacity of nimotuzumab for crossing the blood-brain barrier (BBB) was studied. Immunoscintigraphy done after radiation and nimotuzumab showed a positive $\mathrm{MAb}$ uptake at the known site of the tumors. The patients with complete response showed no antibody accumulation

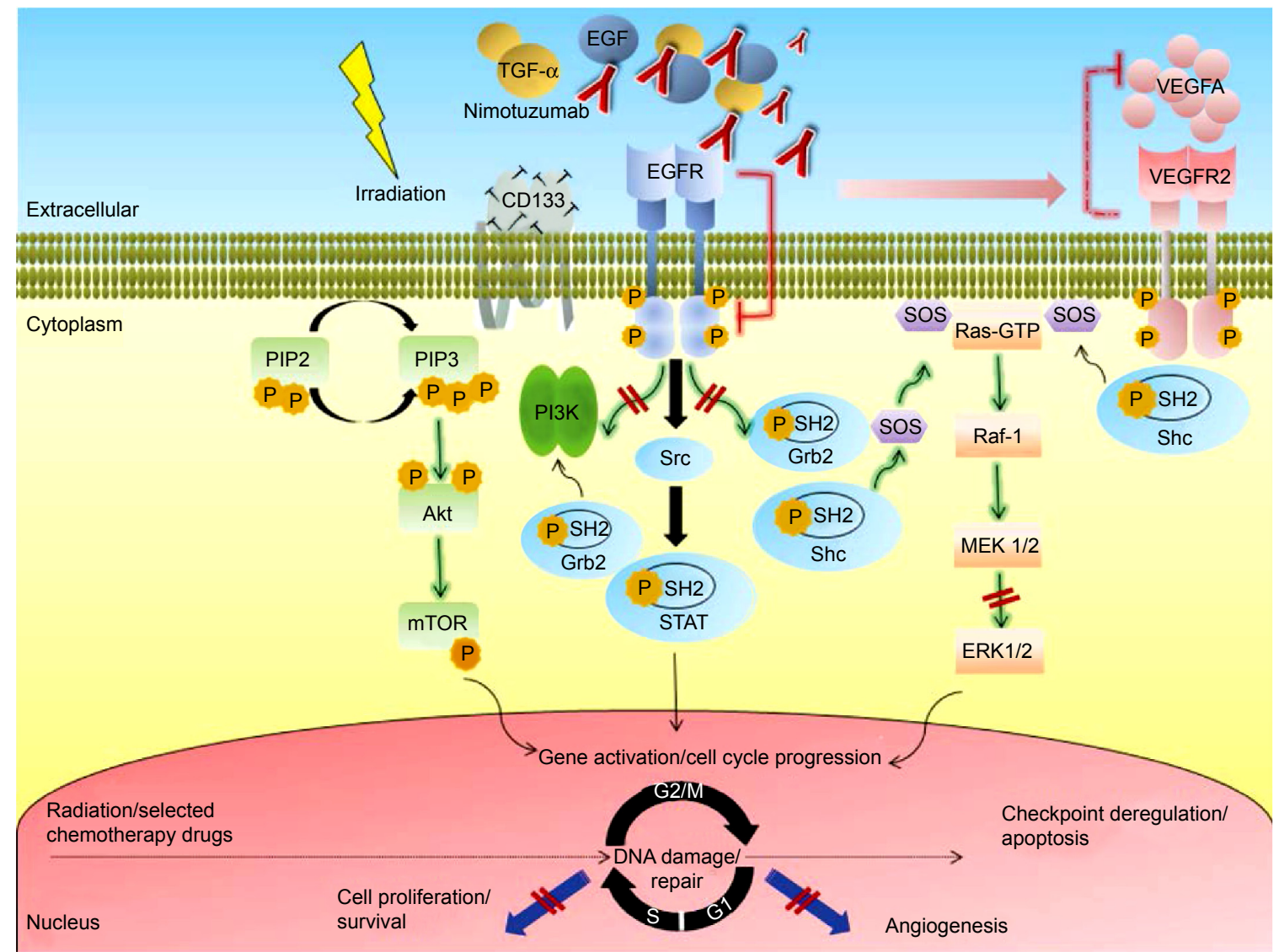

Figure I Simplified schematic illustration depicting different mechanisms implicated in radiosensitization by the antiepidermal growth factor receptor monoclonal antibody nimotuzumab in high-grade glioma.

Note: Copyright (C) 2015. Dove Medical Press. Reproduced from Diaz-Miqueli A, Martinez GS. Nimotuzumab as a radiosensitizing agent in the treatment of high grade glioma: challenges and opportunities. Oncology Targets Therapy. 2013;6:931-942. ${ }^{8}$

Abbreviations: Akt, protein kinase B; CD, cluster differentiation; EGF, epidermal growth factor; EGFR, epidermal growth factor receptor; ERK, extracellular signalregulated kinase; Grb2, growth factor receptor-bound protein 2; GTP, guanosine-5'-triphosphate; MEK, mitogen activated protein kinase kinase; mTOR, mammalian target of rapamycin; PI3K, phosphatidyl inositol 3 kinase; PIP2, phosphatidylinositol 4,5-bisphosphate; PIP3, phosphatidylinositol 3,4,5-trisphosphate; Raf, rapidly accelerated fibrosarcoma; Ras, rat sarcoma; SH2, Src homology-2; shc, Src-homology collagen protein; SOS, son of sevenless homolog I; Src, sarcoma; STAT, signal transducer and activator of transcription; TGF- $\alpha$, transforming growth factor alpha; VEGFA, vascular endothelial growth factor type A; VEGFR2, VEGF receptor 2. 
inside the tumors, while patients showing PR, SD, or progressive disease had a positive uptake of the murine MAb in the known site of the tumor.

Although the BBB may interrupt the delivery of agents to brain malignancies, surgery, radiation, and the tumor itself disrupt its integrity, allowing drug uptake by the tumors. What is more, in the fast-growing glioma, the newly formed blood vessels may lack of BBB function. As a result, nimotuzumab might easily enter the tumor through the intact newly formed blood vessels. ${ }^{11,12}$

\section{A Phase I/II clinical study of concomitant radiochemotherapy with or without nimotuzumab}

A study from the People's Republic of China aimed to determine whether nimotuzumab enhances the effect of radiochemotherapy in malignant glioma. ${ }^{13}$ Forty-one malignant glioma patients were entered in either the treatment group nimotuzumab plus radiochemotherapy or the control group with the placebo and radiochemotherapy. The MST of the treatment and control groups were 16.5 and 10.5 months, respectively. The 1-year OS of the treatment and control groups were $81.3 \%$ and $69.1 \%$, respectively $(P>0.05)$. The objective response rates of the treatment and control groups were $70.0 \%$ and $52.4 \%$, respectively $(P>0.05)$. In conclusion, there was a trend towards improved efficacy of standard therapy when combined with nimotuzumab against glioma.

The study revealed that the mean and median survival times of the patients with malignant glioma were longer in those patients treated with nimotuzumab-combined radiochemotherapy. However, the mean and median survival time and the oneyear survival rate showed no significant difference between the treatment and control groups. The objective response rate between the treatment and control groups also showed no significant difference. It was postulated that the sample size enrolled in the study was too small to detect any statistical difference in median survival time or objective response rate between the treatment and control groups. Although the patient numbers were small, there was a trend toward survival benefit with nimotuzumab and radiochemotherapy.

\section{Phase II trials and clinical experiences} Nimotuzumab alone in pediatric patients with progressive diffuse intrinsic pontine glioma (DIPG)

Patients with clinically and radiologically confirmed, centrally reviewed DIPG, who had failed standard first-line therapy were eligible for this multicenter Phase II trial. ${ }^{14}$ Nimotuzumab (150 $\mathrm{mg} / \mathrm{m}^{2} / \mathrm{wk}$ ), was administered from the first to the seventh week and once every 2 weeks from the eighth to the 18 th week. Response evaluation was based on clinical and MRI assessments. Patients with PR or SD were allowed to continue nimotuzumab. Forty-four patients received at least one dose of nimotuzumab (male/female, 20/24; median age, 6.0 years; range, 3.0-17.0 years). All the patients had received prior radiotherapy. Treatment was well tolerated. Eighteen patients experienced serious adverse events (SAEs). The majority of SAEs were associated with disease progression. Nineteen patients underwent 8 weeks of nimotuzumab: there were two $\mathrm{PR}$, six SD, and eleven progression (PD). Five patients completed 18 weeks of treatment: one of two patients with PR at the eighth week was still PR at the 18th week, and three of six children with SD at the eighth week maintained SD at the 18th week. Time to progression for the four patients with SD or better at the 18th week was 119-335 days. The MST was 3.2 months. Two patients lived 663 and 481 days following initiation of nimotuzumab. Modest activity of nimotuzumab in DIPG, which has been shown previously, was confirmed.

\section{Nimotuzumab in combination with chemotherapy for patients with recurrent malignant glioma}

Our group evaluated the efficiency and side effects of nimotuzumab in combination with chemotherapy for patients with recurrent malignant glioma. ${ }^{15}$ Patients received $200 \mathrm{mg}$ of nimotuzumab infusion intravenously over 60 minutes once weekly for the first 8 weeks and then once every 2 weeks until unacceptable toxicity or tumor progression occurred. Individualized chemotherapy was administered based on O6-methylguanine-DNA methyltransferase (MGMT) expression and previous chemotherapy responses. From December 2008 to April 2010, 14 patients received a total of 122 times of nimotuzumab ranging from 2 to 20 (median, 7.5 times). Combined chemotherapy regimens included: continuous 21-day temozolomide (ten cases), standard 5-day temozolomide (two cases), teniposide plus cisplatin (one case), teniposide plus nimustine (one case). PR and SD were found in three patients (21.4\%) and six patients $(42.9 \%)$, respectively. Disease control rate $(\mathrm{PR}+\mathrm{SD})$ was $64.3 \%$. The median progression-free survival (PFS) was four months (95\% CI: 0.7-7.3), and PFS at 6 months was $30.6 \%$. The most common toxicities included grade I-II neutropenia (two cases), thrombocytopenia (two cases), lymphopenia (one case), nausea and vomiting (three cases), and asymptomatic transaminase increase (one case). One patient developed grade IV neutropenia and thrombocytopenia. One patient developed nimotuzumab-related acneiform rash.

\section{Nimotuzumab in combination with radiotherapy in newly diagnosed HGG patients}

In a single institution experience, ${ }^{16}$ thirty-five patients with HGG were treated with radiation and nimotuzumab. 
Nimotuzumab was administered at $200 \mathrm{mg} / \mathrm{wk}$ for the first 6 weeks combined with radiation followed by a maintenance dose of nimotuzumab every 3 weeks for 1 year. With the median cumulative dose of 2,400 $\mathrm{mg}$, the most frequent toxicities were liver dysfunction, fever, gastrointestinal reaction, anorexia, fatigue, dizziness, and tremors, but none of the above toxicities were severe. The MST was 12.4 months for GBM and 27.0 months for AA patients, longer than that of a matched population treated at the same hospital with radiotherapy alone (median 8.0 and 12.2 months for GBM and AA patients, respectively). Nimotuzumab is a suitable inhibitor with mild and moderate side effects for maintenance.

\section{Nimotuzumab and vinorelbine in combination with radiotherapy for DIPG in childhood}

A pilot Phase II protocol for the evaluation of the combination of nimotuzumab with concomitant radiation and vinorelbine was developed. ${ }^{17}$ A dose of $20 \mathrm{mg} / \mathrm{m}^{2} /$ wk of vinorelbine and a dose of nimotuzumab $150 \mathrm{mg} / \mathrm{m}^{2} /$ wk were administered in the first 12 weeks of treatment, followed by the radiation with 54 Gy from the third to the ninth week. Maintenance of vinorelbine $25 \mathrm{mg} / \mathrm{m}^{2}$ and nimotuzumab was given every 2 weeks until the progression or for up to 2 years. Twentyfive children with a mean age of 7.4 years were entered from August 2009. With the median follow-up time of 29 months, $96 \%$ of children (24/25) presented a response. The toxicity of the combination of these two agents was moderate or mild without acute side effects. $69 \%$ of locally relapsing patients $(11 / 16)$ were reirradiated with 19.8 Gy. 1-year PFS and OS rates were $30 \%$ and $76 \%$, respectively; 2-year OS was 27\%; the median PFS and OS were 8.5 and 15 months, respectively. This strategy seemed to be promising and needs to be confirmed in a large number of cases.

\section{Nimotuzumab in combination with temozolomide and radiation therapy for newly diagnosed GBM patients}

This study was aimed at evaluating the safety and efficacy of a combination of nimotuzumab and standard radiation plus concomitant and adjuvant temozolomide in Chinese patients newly diagnosed with GBM. The study also sought to investigate the possible relationship between EGFR expression and clinical efficacy. ${ }^{18}$ From January 2009 to August 2013, 26 patients with newly diagnosed GBM were entered in the study. All patients received a standard dose of radiation after operation concomitant with $75 \mathrm{mg} / \mathrm{m}^{2} / \mathrm{d}$ of temozolomide from day 1 to day 42, combined with nimotuzumab at a dose of $200 \mathrm{mg} / \mathrm{wk}$ for 6 weeks, followed by six cycles of standard temozolomide $\left(150 \mathrm{mg} / \mathrm{m}^{2}\right.$ for 5 days for the first cycle,
$200 \mathrm{mg} / \mathrm{m}^{2}$ for the next five cycles in each 28 -day cycle). With the immunohistochemistry analysis, EGFR expression in the tumor site was evaluated. Treatment was safe and no severe toxicity was observed. The combination of nimotuzumab with standard treatment including radiation with concomitant and adjuvant TMZ seemed safe and was well tolerated.

In this trial, EGFR expression was detected in 23 patients, but there was no relationship between efficacy of nimotuzumab and EGFR expression. The survival times were similar to those seen in historical data of standard therapy study. ${ }^{2}$ The efficacy of adding nimotuzumab to the standard treatment was modest. With the 6-month PFS of $69.2 \%$ and OS of $88.5 \%$, the median PFS and OS were 10.0 and 15.9 months, respectively. The median PFS was slightly longer than that of patients treated with TMZ plus RT (6.9 months) as Stupp et al reported, ${ }^{2}$ and the median OS did not show a survival advantage (15 months vs 14.6 months). However, it seemed to have a longer median OS for EGFR negative patients (16 months for the EGFR negative group vs 13 months for the EGFR-positive group), but there was no statistical significance. Owing to the small number of 23 patients and the simple positive-negative scoring in the immunohistochemistry method for the evaluation of the EGFR status, which does not account for EGFR overexpression, the study of the correlation of efficacy and EGFR needs to be further investigated. However, a survival effect of nimotuzumab in EGFR-positive patients, especially for patients who may show EGFR overexpression, needs to be paid attention to.

\section{Clinical experience with nimotuzumab ( \pm chemotherapy \pm radiotherapy) in pediatric patients with brain tumors}

An open, prospective clinical study was initiated to analyze the efficacy of treating 22 Cuban patients ${ }^{19}$ with a combination of nimotuzumab with chemotherapy and radiation from December 2005 to December 2007. These patients had been diagnosed with progressive or recurrent brain tumor, classified as high-grade malignant GBM ( $n=6)$, DIPG $(n=6)$, low-grade glioma $(n=4)$, ependymoblastoma $(n=5)$, or thalamic tumor $(\mathrm{n}=1)$. Nimotuzumab was administered in a dose of $100 \mathrm{mg} / \mathrm{wk}$ for 6-8 weeks, and as maintenance treatment every other week until tumor progression. Nimotuzumab was administered as monotherapy or in combination with chemotherapy and/or radiotherapy, and results showed that it was well tolerated in all therapeutic strategies, even with maintenance. Patients developed only mild or moderate side effects such as gastrointestinal reaction, mucositis, and chills. The disease was controlled in 64\% (14/22) of patients; 6-month and 1-year OS were $82 \%$ and $64 \%$, respectively with median OS of 
19 months. Recovery of neurological functions and improvement in general status were notable in patients whose disease was controlled. Nimotuzumab proved to be well tolerated, making it acceptable for chronic use, and implied clinical benefits of survival and functional improvement.

\section{Treatment of children with HGG with nimotuzumab:} a 5-year institutional experience

The purpose of this study was to evaluate the safety and efficacy of nimotuzumab alone or in combination with radiotherapy or chemotherapy in newly diagnosed supratentorial HGG children in Cuba. ${ }^{20}$ According to the treatment stage of each patient at the time of enrollment in the expanded access program, nimotuzumab was administered alone or in combination with the standard 54 Gy radiation, followed by 4-6 cycles of chemotherapy, including procarbazine $100 \mathrm{mg} / \mathrm{m}^{2}$ for 10 days, cyclophosphamide $1 \mathrm{~g} / \mathrm{m}^{2}$ for one day, cisplatin $30 \mathrm{mg} / \mathrm{m}^{2}$ for 4 days, and prednisone $40 \mathrm{mg} / \mathrm{m}^{2}$ for 14 days. From 2007 to 2011, 23 children received $150 \mathrm{mg} / \mathrm{m}^{2} /$ week of nimotuzumab for 12 weeks as induction therapy and every other week as maintenance until tumor progression. The mean age was 12.4 years (range $2-18$ years). Five patients received nimotuzumab in combination with radiotherapy followed by chemotherapy. Sixteen patients who were already irradiated prior to joining the expanded access program received nimotuzumab combined with chemotherapy, and three patients were treated with the antibody alone due to the previously finished radiochemotherapy. The mean number of doses of nimotuzumab administered was 39 (median 19, range 8-106). Similarly to the other studies, nimotuzumab was well tolerated even with maintenance without cumulative toxicity. 20 out of 23 patients were older than 6 years, and many of them could resume school activities during nimotuzumab maintenance. In this trial, the objective response rate was $52.2 \%$, and the disease control rate was $69.5 \%(9 \mathrm{CR}+3 \mathrm{PR}+4 \mathrm{SD})$. The median OS was 32.66 months, and the 1-year OS and 3-year OS were $64 \%$ and $48.8 \%$, respectively. This study confirmed the prolonged administration of nimotuzumab and showed preliminary evidence of clinical benefit in HGG patients with poor prognosis.

\section{Phase III trials}

Radiotherapy plus nimotuzumab or placebo in the treatment of HGG patients

A randomized, prospective, multicentric clinical study was carried out to analyze the combination of radiation with or without nimotuzumab in HGG patients (41 AA and 29 GBM) from June 2005 to June 2010. ${ }^{21}$ Patients received nimotuzumab at a dose of $200 \mathrm{mg} / \mathrm{wk}$ for 6 weeks or placebo combined with radiotherapy in the induction phase. In the maintenance stage, nimotuzumab was given every 3 weeks for 1 year. The median cumulative dose was 3,200 mg, given over a median number of 16 doses. Patients receiving a combination of treatments developed some side effects such as nausea, fever, tremors, anorexia, and liver dysfunction. But in general, the treatment was well tolerated. No anti-idiotypic response was detected, confirming the antibody low immunogenicity. The mean and median survival times for subjects treated with nimotuzumab were 31.06 and 17.76 months versus 21.07 and 12.63 months for the control group. In this randomized trial, nimotuzumab showed an excellent safety profile and significant survival benefit in combination with irradiation in HGG patients.

\section{Phase III study of randomized nimotuzumab therapy in adult GBM patients}

A randomized Phase III study was conducted in Germany in patients with newly diagnosed GBM. ${ }^{22}$ In this study, 149 patients were randomized to receive temozolomide/ radiation with or without nimotuzumab. $75 \mathrm{mg} / \mathrm{m}^{2} / \mathrm{d}$ of temozolomide from day 1 to day 42 was given during radiation, followed by nine cycles of standard temozolomide $\left(150-200 \mathrm{mg} / \mathrm{m}^{2}\right.$ for 5 days in each 28-day cycle). In the nimotuzumab arm, patients received $400 \mathrm{mg}$ of the antibody until week 11 , followed by biweekly doses from week 13 (consolidation phase) for 1 year. MRI evaluations were performed at weeks 12,24 , and 36 by a reference neuroradiologist.

The EGFR-histochemistry, the rate of MGMT-promotermethylation, of EGFR mutant VIII, and of incomplete surgical resection, were similar in both arms. Nimotuzumab was safe and did not show the severe toxicity of standard therapy, including headache, fatigue, vomiting, nausea, thrombocytopenia, aphasia, and mild-to-moderate acneiform rash. Although the median OS of patients in both arms was not significantly different (22.3 months [CI: 17.2-26.5] receiving nimotuzumab vs 19.6 months [CI: 14.8-24.0] in the control arm), there were significant differences with reference to the subgroups with nonmethylated MGMT and EGFR-positive glioblastoma. The median OS was 23.8 months in comparison with 13.8 months in the control group ( $P=0.03)$. Therefore, future studies on the efficacy of nimotuzumab should focus on patients with nonmethylated MGMT and EGFR-positive glioblastoma that present with residual tumor after surgery.

\section{Conclusion}

In conclusion, nimotuzumab has unique functional properties, unlike other anti-EGFR antibodies. Compared with other 
EGFR target drugs, the efficacy of nimotuzumab seems to be true. It is a very well-tolerated drug, and has an encouraging safety profile in the treatment of HGG with or without radiotherapy and chemotherapy. Emerging trials suggested nimotuzumab had moderate efficacy in treating HGG, particularly in the subgroups of pediatric DIPG, nonmethylated MGMT, and EGFR-positive GBM with residual tumor. In our opinion, it is critical to detect context-dependent oncogene addiction, which may affect the clinical response for nimotuzumab. Because of the complexity of the signaling network inducing the crosstalk and feedback problem, a suitable combination treatment including nimotuzumab and other target drug maybe necessary for HGG to suppress resistance. As a result, multiple center randomized controlled Phase III clinical trials need to be conducted to confirm the efficacy and toxicity of nimotuzumab in HGG.

\section{Disclosure}

The authors report no conflicts of interest in this work.

\section{References}

1. Stupp R, Mason WP, van den Bent MJ, et al. Radiotherapy plus concomitant and adjuvant temozolomide for glioblastoma. $N$ Engl J Med. 2005;352:987-996.

2. Stupp R, Hegi ME, Mason WP, et al. Effects of radiotherapy with concomitant and adjuvant temozolomide versus radiotherapy alone on survival in glioblastoma in a randomised phase III study: 5-year analysis of the EORTC-NCIC trial. Lancet Oncol. 2009;10(5):459-466.

3. Jarvela S, Helin H, Haapasalo J, et al. Amplifcation of the epidermal growth factor receptor in astrocytic tumours by chromogenic in situhybridization: association with clinicopathological features and patient survival. Neuropathol Appl Neurobiol. 2006;32:441-450.

4. Viana-Pereira M, Lopes JM, Little S, et al. Analysis of EGFR overexpression, EGFR gene amplifcation and the EGFRvIII mutation in Portuguese high-grade gliomas. Anticancer Res. 2008;28:913-920.

5. Mazzoleni S, Politi LS, Pala M, et al. Epidermal growth factor receptor expression identifies functionally and molecularly distinct tumorinitiating cells in human glioblastoma multiforme and is required for gliomagenesis. Cancer Res. 2010;70(19):7500-7513.

6. Mellinghoff IK, Wang MY, Vivanco I, et al. Molecular determinants of the response of glioblastomas to EGFR kinase inhibitors. $N$ Engl J Med. 2005;353:2012-2024.

7. Ramakrishnan MS, Eswaraiah A, Crombet T, et al. Nimotuzumab, a promising therapeutic monoclonal for treatment of tumors of epithelial origin. MAbs. 2009;1(1):41-48.
8. Diaz-Miqueli A, Martinez GS. Nimotuzumab as a radiosensitizing agent in the treatment of high grade glioma: challenges and opportunities. Onco Targets Ther. 2013;6:931-942.

9. Bode U, Massimino M, Bach F, et al. Nimotuzumab treatment of malignant gliomas. Expert Opin Biol Ther. 2012;12(12):1649-1659.

10. Ramos TC, Figueredo J, Catala M, et al. Treatment of high-grade glioma patients with the humanized anti-epidermal growth factor receptor (EGFR) antibody h-R3: report from a phase I/II trial. Cancer Biol Ther. 2006;5(4):375-379.

11. Qin D, Ou G, Mo H, et al. Improved efficacy of chemotherapy for glioblastoma by radiation-induced opening of blood-brain barrier: clinical results. Int J Radiat Oncol Biol Phys. 2001;51:959-962.

12. Ramos-Suzarte M, Rodriguez N, Oliva JP, et al. $99 \mathrm{mTc}$-labeled antihuman epidermal growth factor receptor antibody in patients with tumours of epithelial origin, part II: clinical trials safety and diagnostic efficacy. J Nucl Med. 1999;40:768-775.

13. Hong J, Peng Y, Liao Y, et al. Nimotuzumab prolongs survival in patients with malignant gliomas: a phase I/II clinical study of concomitant radiochemotherapy with or without nimotuzumab. Exp Ther Med. 2012;4(1):151-157.

14. Bartels U, Wolff J, Gore L, et al. Phase 2 study of safety and efficacy of nimotuzumab in pediatric patients with progressive diffuse intrinsic pontine glioma. Neuro Oncol. Epub May 20, 2014.

15. Yang QY, Shen D, Sai K, et al. [Nimotuzumab in combination with chemotherapy for patients with malignant gliomas]. Zhonghua Zhong Liu Za Zhi. 2011;33(3):232-235. Chinese.

16. Solomon MT, Miranda N, Jorrín E, et al. Nimotuzumab in combination with radiotherapy in high grade glioma patients: a single institution experience. Cancer Biol Ther. 2014;15(5):504-509.

17. Massimino M, Biassoni V, Miceli R, et al. Results of nimotuzumab and vinorelbine, radiation and re-irradiation for diffuse pontine glioma in childhood. J Neurooncol. 2014;118(2):305-312.

18. Wang Y, Pan L, Sheng XF, et al. Nimotuzumab, a humanized monoclonal antibody specific for the EGFR, in combination with temozolomide and radiation therapy for newly diagnosed glioblastoma multiforme: first results in Chinese patients. Asia Pac J Clin Oncol. Epub January 24, 2014.

19. Saurez G, Cabanas R, Zaldívar M, et al. Clinical experience with nimotuzumab in cuban pediatric patients with brain tumors, 2005 to 2007.MEDICC Rev. 2009;11(3):27-33.

20. Cabanas R, Saurez G, Rios M, et al. Treatment of children with high grade glioma with nimotuzumab: a 5-year institutional experience. MAbs. 2013;5(2):202-207.

21. Solomón et al. Radiotherapy plus nimotuzumab or placebo in the treatment of high grade glioma patients: results from a randomized, double blind trial. BMC Cancer. 2013;13:299.

22. Westphal M, Bach F. Final results of a randomized phase III trial of nimotuzumab for the treatment of newly diagnosed glioblastoma in addition to standard radiation and chemotherapy with temozolomide versus standard radiation and temoziolamide. ASCO Annual Meeting; June 1-5, 2012; Chicago, IL, USA. J Clin Oncol. 30(Suppl):Abstr 2033.
OncoTargets and Therapy

\section{Publish your work in this journal}

OncoTargets and Therapy is an international, peer-reviewed, open access journal focusing on the pathological basis of all cancers, potential targets for therapy and treatment protocols employed to improve the management of cancer patients. The journal also focuses on the impact of management programs and new therapeutic agents and protocols on

\section{Dovepress}

patient perspectives such as quality of life, adherence and satisfaction. The manuscript management system is completely online and includes a very quick and fair peer-review system, which is all easy to use. Visit http://www.dovepress.com/testimonials.php to read real quotes from published authors. 